\title{
Dynamic and complex transcription factor binding during an inducible response in yeast
}

\author{
Li Ni, ${ }^{1,9}$ Can Bruce, ${ }^{2,3,9}$ Christopher Hart, ${ }^{4}$ Justine Leigh-Bell, ${ }^{5}$ Daniel Gelperin, ${ }^{6}$ Lara Umansky, ${ }^{1}$ \\ Mark B. Gerstein, ${ }^{2,7,8}$ and Michael Snyder ${ }^{1,2,10}$ \\ ${ }^{1}$ Department of Molecular, Cellular, and Developmental Biology, Yale University, New Haven, Connecticut 06520, USA; \\ ${ }^{2}$ Department of Molecular Biophysics and Biochemistry, Yale University, New Haven, Connecticut 06520, USA; ${ }^{3}$ Keck \\ Foundation Biotechnology Resource Laboratory, Yale University, New Haven, Connecticut 06520, USA; ${ }^{4}$ Helicos Biosciences, \\ Cambridge, Massachusetts 02139, USA; ${ }^{5}$ Center for Regulation Genomics, Barcelona, Spain; ${ }^{6}$ Affomix Corporation, New Haven, \\ Connecticut 06511, USA; ${ }^{7}$ Department of Computer Science, Yale University, New Haven, Connecticut 06520, USA; ${ }^{8}$ Program \\ in Computational Biology and Bioinformatics, Yale University, New Haven, Connecticut 06520, USA
}

\begin{abstract}
Complex biological processes are often regulated, at least in part, by the binding of transcription factors to their targets. Recently, considerable effort has been made to analyze the binding of relevant factors to the suite of targets they regulate, thereby generating a regulatory circuit map. However, for most studies the dynamics of binding have not been analyzed, and thus the temporal order of events and mechanisms by which this occurs are poorly understood. We globally analyzed in detail the temporal order of binding of several key factors involved in the salt response of yeast to their target genes. Analysis of Yap4 and Sko1 binding to their target genes revealed multiple temporal classes of binding patterns: (1) constant binding, (2) rapid induction, (3) slow induction, and (4) transient induction. These results demonstrate that individual transcription factors can have multiple binding patterns and help define the different types of temporal binding patterns used in eukaryotic gene regulation. To investigate these binding patterns further, we also analyzed the binding of seven other key transcription factors implicated in osmotic regulation, including Hot1, Msn1, Msn2, Msn4, Skn7, and Yap6, and found significant coassociation among the different factors at their gene targets. Moreover, the binding of several key factors was correlated with distinct classes of Yap4- and Sko1-binding patterns and with distinct types of genes. Gene expression studies revealed association of Yap4, Sko1, and other transcription factor-binding patterns with different gene expression patterns. The integration and analysis of binding and expression information reveals a complex dynamic and hierarchical circuit in which specific combinations of transcription factors target distinct sets of genes at discrete times to coordinate a rapid and important biological response.
\end{abstract}

[Keywords: Salt response; stress; transcription factor; yeast]

Supplemental material is available at http://www.genesdev.org.

Received January 14, 2009; revised version accepted April 10, 2009.

The execution of biological processes involves the careful orchestration of complex molecular events. A detailed understanding of the regulation of these processes will require elucidating the entire suite of interactions that occur in a dynamic fashion at a variety of levels, including transcriptional, post-transcriptional, and translational.

To date, large-scale studies have primarily analyzed the dynamics of expression patterns during developmental and environmental induction responses (Hamatani et al. 2004; Berry and Gasch 2008). These studies have revealed

\footnotetext{
${ }^{9}$ These authors contributed equally to this work.

${ }^{10}$ Corresponding author.

E-MAIL Michael.snyder@yale.edu; FAX (203) 432-6161.

Article is online at http://www.genesdev.org/cgi/doi/10.1101/gad.1781909.
}

changes in mRNA levels for thousands of genes. However, how the regulation of expression of these thousands of genes is executed is not known. In particular, the transcription factors that regulate genes, the dynamics of their binding patterns, and the complexities of which factors work together to regulate which genes cannot easily be deduced from such studies. Most experiments to examine the dynamics of transcriptional regulation have focused on one or a few regulators. Alternatively, other studies have globally mapped the suite of binding sites of transcription factors, usually under a single condition or time point (Harbison et al. 2004). Such global studies have provided insight into which factors work together to regulate which genes (Reed et al. 2008). However, they usually do not follow the temporal order of events that 
mediate the dynamics of gene expression. To address this problem, several studies have integrated binding information with expression data or other information to deduce expression dynamics (Simon et al. 2001; Luscombe et al. 2004). However, these studies do not directly follow the binding dynamics, and thus the types of binding patterns that exist and the dynamics of how factors come together to regulate sets of genes is usually not known.

One system that has been explored in a temporal fashion is the Drosophila visceral muscle development in which the binding of factors to large numbers of genes has been investigated (Jakobsen et al. 2007). Analysis of this system has revealed the binding of combination of transcription factors to distinct sets of genes for a limited number of factors, and has suggested a complex organization of transcription factor binding to distinct targets. However, other types of cellular responses such as environmental challenges have not been examined. Analysis of such systems is important because such processes are reversible and therefore may use different strategies.

We analyzed the salt stress response of yeast as a valuable model for examining highly regulated and rapid pathways controlling complex biological processes. The salt response is a well-characterized pathway in which the kinetics of RNA expression have been thoroughly investigated (Berry and Gasch 2008). The process involves a large number of transcription factors, some of which are specifically involved in the osmotic stress response (Msn1, Smp1, and Hot1), and others of which have been shown to be important in general stress responses (Rep et al. 1999; Gasch et al. 2000; de Nadal et al. 2003). We followed the kinetics of binding of several key factors and found that different binding patterns are often evident for the same transcription factor. By mapping binding of a large suite of factors we determined that many of the factors work together in a dynamic fashion to control common gene targets and specific types of genes are controlled by distinct combination of factors. Our results demonstrate that a complex set of regulatory interactions occur to form a dynamic regulatory code responsible for controlling a highly conserved process in eukaryotes.

\section{Results}

Distinct classes of dynamic binding revealed by chromatin immunoprecipitation (ChIP) chip of Yap4 and Sko1

We wished to examine the dynamics of the transcription factor binding during the salt response of yeast. We first examined the binding of five factors, Yap4, Sko1, Yap6, Msn2, and Skn7 after incubation with high salt $(0.6 \mathrm{M})$ for $30 \mathrm{~min}$ and also in the absence of salt using ChIP chip (Horak et al. 2002; Borneman et al. 2007a). Thirty minutes is an optimal timepoint for observing maximal numbers of changes in both binding events and gene expression patterns as described below. Yeast strains in which the transcription factors were epitope-tagged with 13 copies of c-myc at their $\mathrm{C}$ terminus (13X c-myc) were incubated in the presence or absence of salt. Formaldehyde was added to cross-link protein to DNA, and the cells were harvested, the DNA sonicated, and the transcription factor-bound DNA recovered by immunoprecipitation using anti-c-myc antibodies. The DNA was labeled and used along with control DNA to probe highdensity Nimblegen arrays that contain probes every 50 base pairs (bp) (Borneman et al. 2007a,b). After normalizing data, peaks were scored by using a program implementing the same algorithm as Tilescope (Zhang et al. 2007), and binding sites that lay within $1 \mathrm{~kb}$ or closer were assigned to their associated gene (see the Materials and Methods). Several binding patterns emerged. Some transcription factors, such as Msn2 and Msn4, showed little or no evidence of binding prior to incubation of salt and bound many targets after $30 \mathrm{~min}$. This result is expected since these factors are cytosolic and translocate to the nucleus upon stimulation with salt (Gorner et al. 1998; Chi et al. 2001; Mayordomo et al. 2002). However, other factors (Yap4, Yap6, and Sko1) bound to many targets under both conditions although for each factor a twofold to fourfold increase in targets was observed at 30 min (Supplemental Fig. S1; Supplemental Table S1).

The association of Yap4 and Sko1 at targets in both the absence of salt and after incubation with salt prompted us to examine the binding of these factors in more detail. ChIP chip was performed for Yap4- and Sko1-tagged cells incubated in the presence of salt for 5, 15, 30, and $45 \mathrm{~min}$, and in the absence of salt. Signal maps revealed complex patterns of binding (Fig. 1A-C). Targets were scored as above, and 30 were validated at each time point using quantitative PCR (qPCR) (Supplemental Fig. S2). The patterns of binding were classified according to the temporal pattern of binding signal using a penalized and weighted K-means clustering approach (Fig. 2; Tseng 2007). For each factor three major patterns of binding were observed. For Yap4, the major categories were (1) Class 1, constant binding at all time points and in the absence of salt; (2) Class 2, rapid induction reaching a plateau at 15 min after treatment with salt; and (3) Class 3 , induction initiated after a 5-min delay, reaching a plateau at $30 \mathrm{~min}$ (Fig. 2). In addition, minor binding patterns were observed in which a few binding sites exhibited either a transient increase (Class 4) or decrease (Class 5) in binding. For Sko1, the major categories were (1) Class 1, a constant binding pattern; (2) Class 2, a gradual increase in binding; and (3) Class 3, a rapid and transient increase in binding (Fig. 2). A few binding sites exhibited a slower increase binding. These results demonstrate that a variety of distinct binding patterns occur for individual transcription factors, and that different factors can have different patterns of binding.

\section{Analysis of other factors that may associate with Yap4} and Sko1

The fact that multiple binding patterns exist for Yap4 and Skol suggests that additional factors likely help mediate their binding. We therefore attempted to determine whether other factors preferentially coassociate with 
A

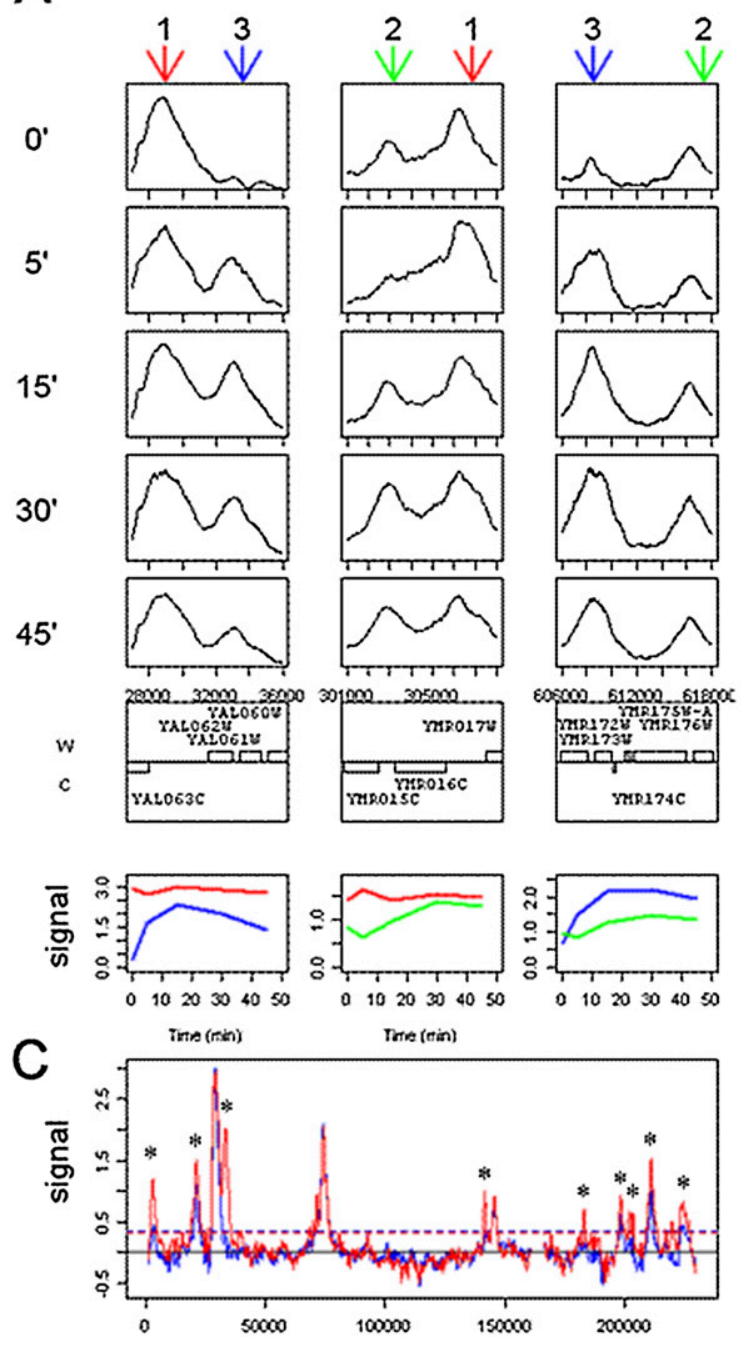

B
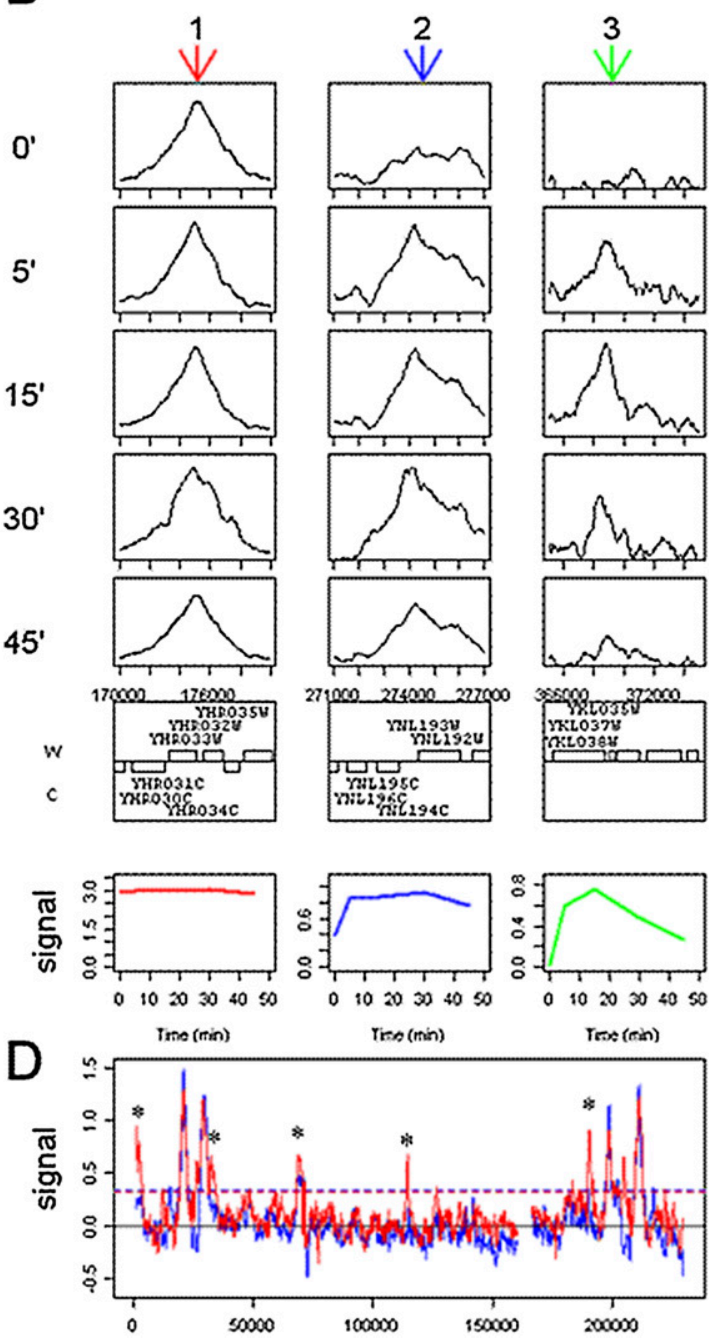

Figure 1. Signal map of the ChIP chip data showing representative examples of peaks that changes during the stress response for Yap4 $(A)$ and Skol $(B)$ (see Fig. 2 for the different patterns). Within each panel, the signal around the chromosomal location indicated by the vertical colored arrow on top is shown for times $0,5,15,30$, and $45 \mathrm{~min}$ post-induction. The ORFs for that chromosomal location are shown below the bottom signal map for each set (for clarity, only those nearest the signal peaks are labeled). The colored curves in the bottom graphs summarize the signal at the location indicated by the colored arrows. $C$ and $D$ show the binding signal for Yap4 and Sko1, respectively (blue, at $0 \mathrm{~min}$; red, at $30 \mathrm{~min}$ ), on chromosome 1 . Dashed line shows the FDR $=0.01$ threshold, stars indicate locations where binding is different at 30 min relative to $0 \mathrm{~min}$.

Yap4 and Sko1, and whether this association varies with the different binding classes. We determined the binding targets of seven other transcription factors previously implicated in the salt response using epitope-tagged strains and ChIP chip. The factors examined were Hotl, Msn1, Msn2, Msn4, Skn7, Smp1, and Yap6 and were chosen because at the time this study was initiated these factors were shown through mutational and/or expression studies to have a role or likely to have a role in stress responses (Mendizabal et al. 1998; Rep et al. 1999; Zahringer et al. 2000; Pascual-Ahuir et al. 2001; Hohmann 2002; de Nadal et al. 2003; Nevitt et al. 2004). Some of these factors (Sko1, Skn7, Yap6, Msn2, and Msn4) have been analyzed previously by ChIP chip either in rich medium or stress conditions (albeit not usually high salt)
(Harbison et al. 2004; Proft et al. 2005; Tan et al. 2008); in our study we analyzed them using the same high salt conditions and strains and with high-density tiling arrays. We examined cells 30 min post-salt induction; at this time dynamic changes were observed for Yap4 and Sko1. As summarized in Figure 3, extensive overlap was observed between Yap4 and Skol. In addition many other factors often bound the same targets including different combinations of Skn7, Smp1, Msn2, Sko1, Yap6, and Yap4 (Fig. 3A). Particularly correlated were Yap4-Yap6, Sko1-Yap6, Msn2-Sko1, Msn2-Skn7, and Skn7-Yap6, in which $>50 \%$ of the targets of one factor overlap with the other; for Yap4 and Yap6, 73\%-86\% of the Yap6 targets (depending on the time point) overlap with Yap4. Although a few of these associations had been noted 
Ni et al.

A
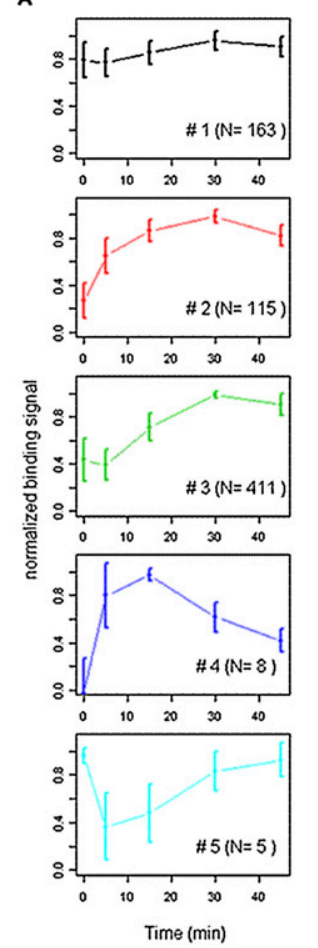

Figure 2. Binding time-course patterns of ChIP chip signal for Yap4 and Sko1. The binding signals were normalized to a maximum of 1.0 before statistical clustering, as detailed in the Materials and Methods, to obtain the groups shown here. Error bars show standard deviation.

previously such as Yap4-Yap6, most of the associations we find are novel; this may be due to the high quality of the data generated in this study using the high-density arrays. Overall, these results suggest that the different factors often function with one another to regulate the same targets.

We next determined if binding of any of the factors correlate with the distinct binding patterns of Yap4 and Sko1 (Fig. 3B). Many Yap4 constitutively bound genes (Class 1) were bound by Skol and Yap6 both at 0 min and $30 \mathrm{~min}$ after salt induction relative to that expected at random. Interestingly, Msn2 also showed preferential binding at $30 \mathrm{~min}$ to the constitutive Yap4-binding targets (Class 1), and is presumably induced during the activation of the response. We also found that Msn2 preferentially binds inducible (Class 2) Yap4-binding targets relative that expected at random. We also found that Yap6 and Sko1 bind a significant number of saltinduced Yap4-binding targets (Class 2) at $30 \mathrm{~min}$; these targets are not bound at $0 \mathrm{~min}$. These results suggest that both constitutive and inducible binding targets are bound by these pairs of factors. Thus, a number of associations appear with Yap4 during both constitutive binding (Class 1) and the salt-induced response (Class 2).

We also examined whether different types of Yap4 sites exhibited differences in nucleosome occupancy using the data of Lam et al. (2008). We found that rapidly bound Yap4 sites (Class 2) and those with delayed Yap4 binding
(Class 3) were both relatively unoccupied in untreated cells $(-0.55 \pm 0.33$ and $-0.69 \pm 0.42$, respectively). These results indicate that both sites are poised for binding, and that the delay in binding is not likely to be due to the need to remove nucleosomes.

As observed for Yap4, many of the constitutive binding targets (Class 1) for Sko1 were already bound at 0 min by Yap4, Yap6, and Sko1 itself. These results suggest that these gene targets were already bound by salt-responsive factors and maintain binding upon salt treatment. In addition, some of the 30-min inducible Skol-binding targets (Class 2) were also bound by Smp1. Overall, these different results indicate that many Yap4- and Sko1bound targets are already enriched for binding by other salt-responsive transcription factors. Nonetheless, the binding of other factors correlates with induced binding, suggesting that binding of these different components at induced targets regulates gene expression.

\section{Motif analysis indicates a strong correlation of binding patterns}

In addition to correlation of binding patterns of other factors, we also examined motifs that were associated with Yap4, Yap6, and Sko1 (Fig. 4). As expected, we found motifs for the binding sites themselves (e.g., Yap4 and Yap6, which have a similar consensus binding site and Sko1). Consistent with the association data we also found Sko1, Yap4, and Yap6 motifs associated with one another, indicating the factors often work together. We also found a significant correlation of motifs of other factors with each of these factors. In particular, we found Skn7 associated with Yap4, and Msn2 and Msn4 associated with Sko1 and Yap6; these data are also consistent with the association studies. Finally, we also found correlations with a variety of other factors not analyzed in this study, many of which were not known previously to be involved in the salt response (e.g., Cha4, Pdr3, Pho4, Sfp1, and Ume6). These results indicate that other factors are likely involved in the salt response, and may have roles beyond those previously known.

Distinct classes of genes are targets of Yap4, Yap6, and Sko1

Analysis of the types of genes bound by Yap4 and Sko1 using the Gene Ontology (GO) classification system and GO Term Finder revealed overlapping but distinct functional classes of genes bound by these factors (Fig. 5A,B). The two factors bound genes involved in hexose transport and glucose and ethanol catabolism. In addition, Yap4 targets were enriched for oxidoreductase activity, whereas Skol targets were enriched for glycolysis. Yap6 targets are enriched for many of the same categories and also some distinct categories. For example, the ribosomal protein genes are preferential targets of Yap6. Of particular interest are the inducible categories of genes. The oxidoreductase and glucose and alcohol catabolic genes (bound by Yap4) and structural components of the ribosome (bound by Yap6) are examples of types of genes at which binding is induced by specific factors. 
A

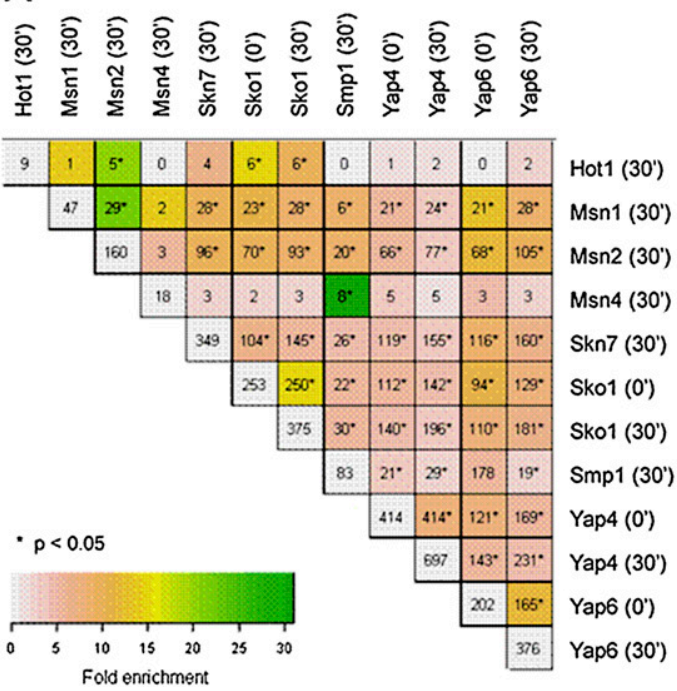

B
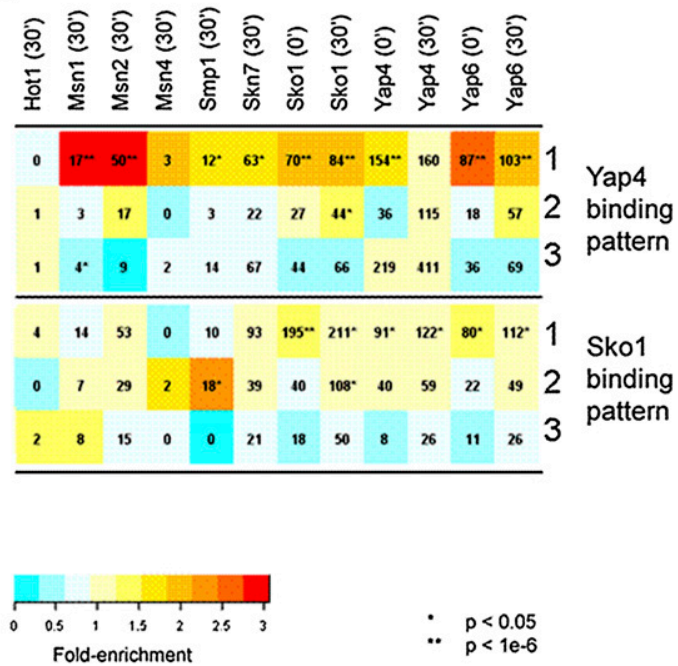

$p<0.05$

Figure 3. (A) Number of promoters bound by pairs of transcription factors and times as indicated. When members of a pair are identical, the given number is the number bound by that transcription factor only. The numbers given for Yap4 and Sko1 are highconfidence sites within promoters after keeping only those binding sites that were identified in two consecutive time points after salt induction. Numbers with a star are significantly $(P=0.05)$ higher than expected for a random segregation of transcription factor-binding sites among promoters. The color scale indicates how many fold greater than expected is the enrichment factor. $(B)$ Number of promoters showing the YAP4-binding patterns (top panel) and Skol-binding patterns (bottom) (see Fig. 2), and bound by the indicated transcription factors at the indicated time points. Combinations with one or two stars show significant enrichment $(P<0.05$ and $1 \mathrm{e}-6)$ relative to that expected for random assortment. The color scale indicates the fold enrichment.

\section{Different combinations of factors regulate types} of genes

We also examined the types of genes regulated by the different combinations of transcription factors using GO
Term Finder analysis (Fig. 5). Many of the combinations bound to the same categories of genes as above. For example, Sko1-Yap4, Sko1-Yap6, and Yap4-Yap6 often bind upstream of transmembrane transporters, and Skn7-Yap6 and Smp1-Yap6 often cobind at genes
Yap6

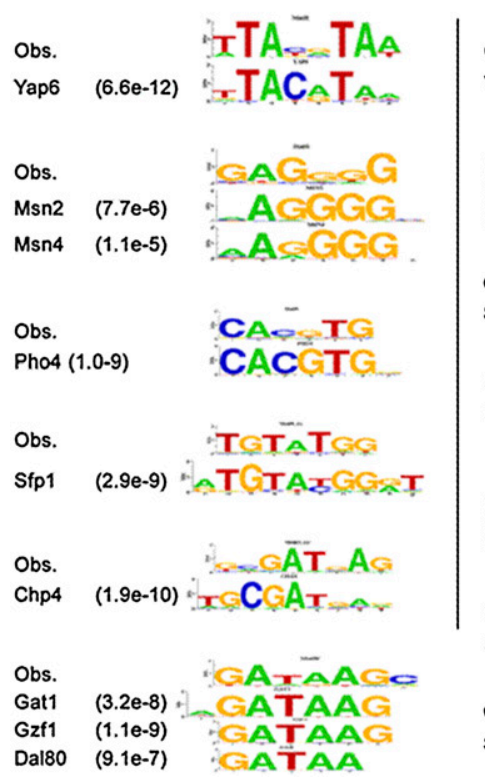

Yap4

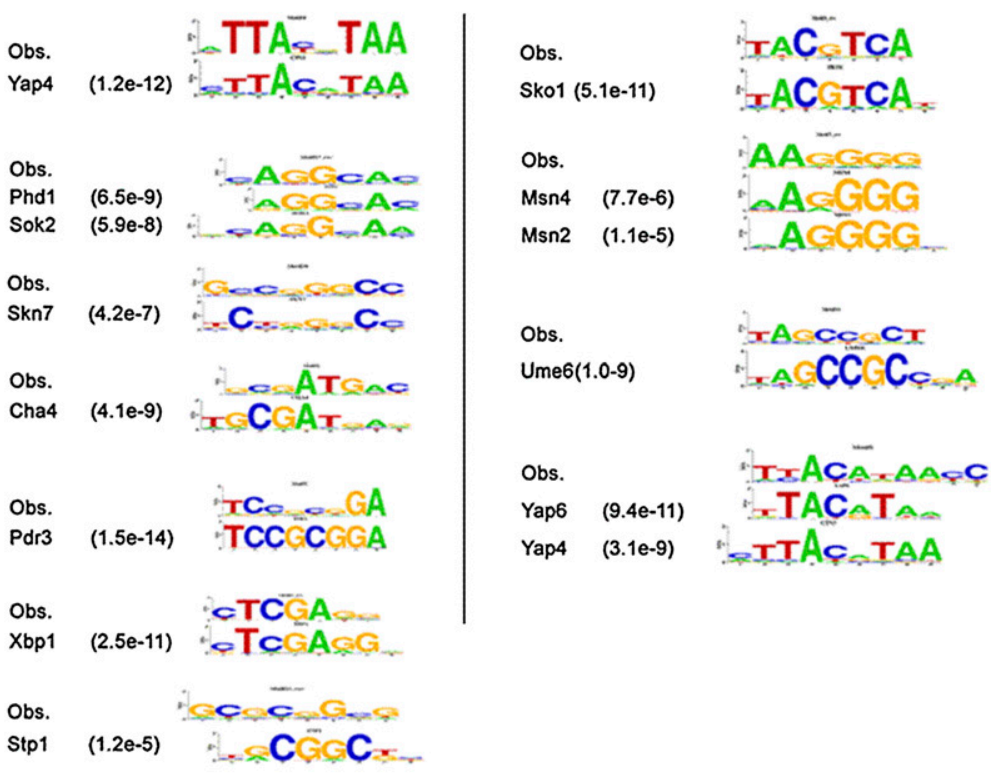

Figure 4. Motifs found to be significantly enriched within promoters bound by Yap4, Yap6, and Sko1. Below each motif are shown known motifs that match this motif along with the significance of the match. See the Materials and Methods for details. 


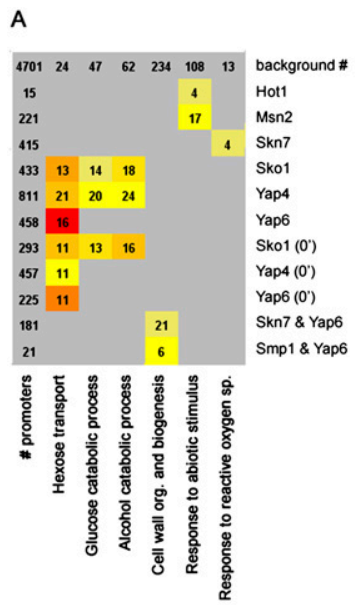

B

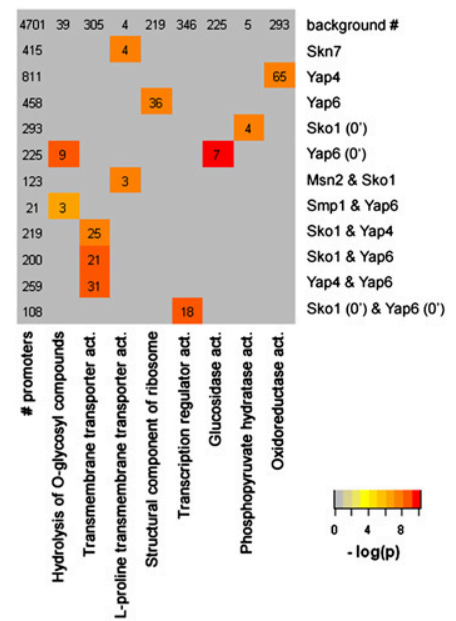

Figure 5. Enrichment for GO functional categories $(A)$ or GO process categories $(B)$ among genes promoters bound by the indicated transcription factors or pairs of them. Binding times are for $30 \mathrm{~min}$ post-induction unless indicated otherwise $(0 \mathrm{~min})$. For single transcription factors, only those that show significant $(P<0.01)$ GO category enrichment are shown and are colorcoded for their significance level. Only number of genes with a significant $(P<0.01)$ enrichment for a GO functional category are shown and are color-coded for their significance level. For pairs of transcription factors, only those genes with enrichment for GO categories that occur in neither member of the pair by itself are shown. The first row ("background \#") shows the number of genes among the total considered $(n=4707$ ORFs of status "verified" or "uncharacterized") belonging to the indicated GO category. The first column shows the number of promoters among the 4707 that are bound by the indicated transcription factor(s) at the indicated time point.

encoding constituents of the cell wall. These results suggest that eukaryotes use combinations of transcription factor to regulate distinct classes of genes.

Genes whose promoters were bound by certain pairs of transcription factors were enriched by different GO categories at $0 \mathrm{~min}$ versus $30 \mathrm{~min}$ (Fig. 5). For example, the Sko1-Yap4, Sko1-Yap6, and Yap4-Yap6 pairs each bind genes involved in transmembrane transporter activity at $30 \mathrm{~min}$. A significant number of genes encoding proteins identified as "transcription regulator(s)" are bound by Sko1 and Yap6 before induction but not after induction; this is due to the number of induced genes that makes the proportion of transcription factors insignificant. Overall these differences indicate the physiological changes that take place as the cell adjusts to the salt stress and that transcription factor combinations that are responsible for these changes.

\section{Correlation of binding classes with gene expression}

We next examined how the binding information correlated with gene expression patterns in wild-type and mutant strains. Briefly, wild-type cells were treated with salt and harvested at $0,7,15,22,30,45$, and $60 \mathrm{~min}$, poly $(\mathrm{A})^{+}$RNA was prepared and used to probe yeast oligonucleotide arrays (see Materials and Methods). The data were scored and gene expression patterns were analyzed. Statistical clustering analysis of genes showing variable expression levels after salt induction revealed three major patterns of gene expression (Fig. 6A): (1) constitutively expressed genes (2) genes whose expression increased at $\sim 15-30 \mathrm{~min}$ and then return to the levels of unstressed cells by 45-60 min, and (3) those whose expression levels decreased at around 15-30 min and then return to wild-type levels after $45-60 \mathrm{~min}$. For the transiently regulated genes, it was observed that there was a delay in their response sometimes.

Analysis of the types of genes in each expression class using the GO classification system and GO Term Finder (at the Saccharomyces cerevisiae Database Web site) revealed a significant enrichment in genes involved in stress response, metabolism, and proteolysis in the category of genes whose expression transiently increases (Class 2 genes); sporulation genes are also induced; we presume that reflects the transfer to stress conditions. Expression of ribosomal structural and processing components and chromosomal proteins is deceased after the addition of salt (Supplemental Table S2). For the most part, these patterns have been observed previously (Berry and Gasch 2008).

For each of the binding time-course patterns, constant, rapid rise, and slow rise, we analyzed the expression patterns. Surprisingly, we found that each binding timecourse cluster contained the major patterns of gene expression: constant, transiently increased, or decreased. However, the frequency varies for the different binding time-course clusters. For Yap4 targets that show a rapid increase in binding (Class 2), there is a greater incidence of transient increase in expression than a decrease, as expected. For the Yap4 targets whose Yap4 binding slowly increases (Class 3) there is a modest enrichment for genes whose expression is unchanged, and for Yap4 targets whose binding is unchanged (Class 1), there is a slight increase in gene expression. This latter observation is with the binding of other factors such as Msn2 at some of the targets (see below). Overall, these results suggest that for many genes the binding of Yap4 correlates with activation of gene expression. For Sko1, no significant enrichment was observed between binding and gene expression. These results suggest that binding by itself may not regulate gene expression, but rather that regulation occurs in conjunction with the presence of other factors. Consistent with this hypothesis, Skol is preferentially bound at targets whose expression is transiently induced; even though its presence is constitutive (Fig. 6B).

To gain further insight into which factors might facilitate regulation of gene expression during the salt response we examined the binding patterns of each of the factors. Hot1, Msn2, Sko1, and Yap6 are enriched with the transient increase in gene expression, whereas $\mathrm{Skn} 7$, Yap4, and Yap6 enrich with a transient decrease in gene expression. The observation that some factors are bound at both induced and repressed genes (notably Yap6), indicates that these regulators can have both a positive and a negative role in gene expression. Positive and 
A

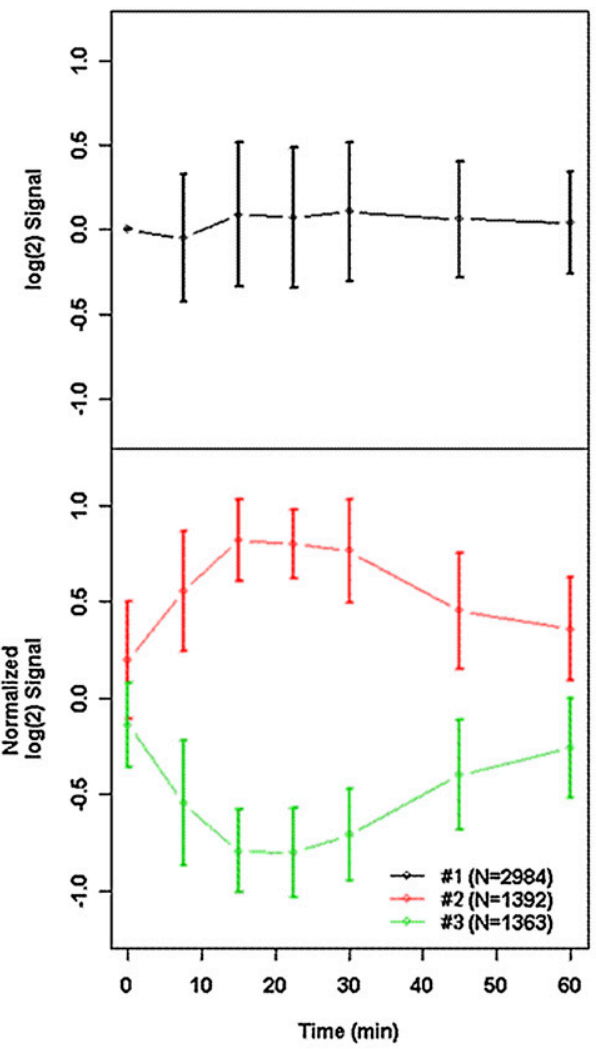

B

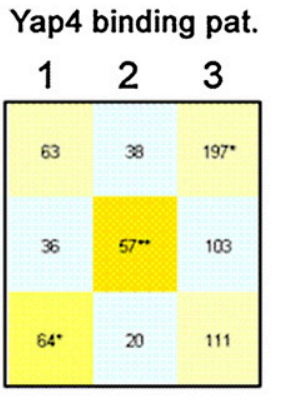

Sko1 binding pat.

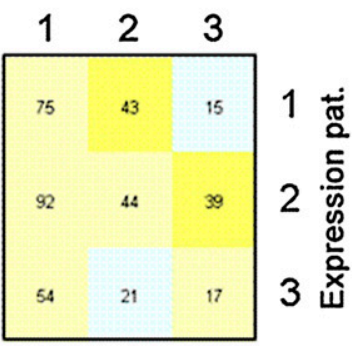

C
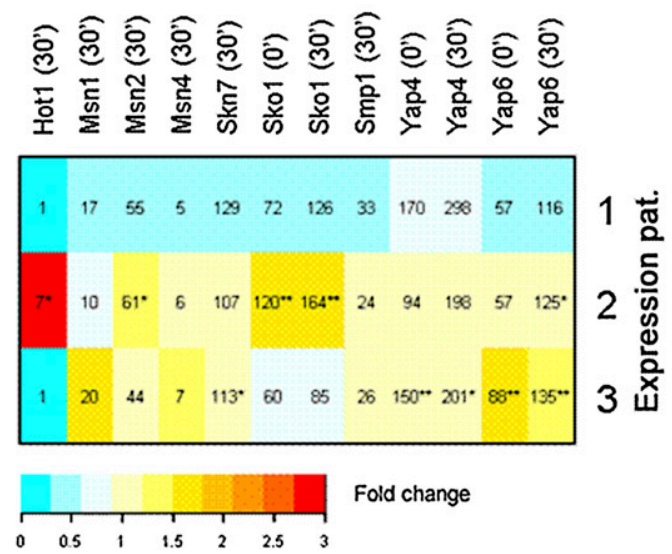

Figure 6. (A) The three main expression patterns following salt induction. (Top panel) Mean and standard deviation of fold change in expression (on a $\log [2]$ scale, normalized to 0 at $t=0 \mathrm{~min}$ ) for expression pattern \#1, where the difference between the greatest and least value is $<0.5 \mathrm{U}$. (Bottom panel) Mean and standard deviation of normalized fold change in expression for patterns \#2 (red) and \#3 (green); For illustrative purposes pattern \#2 is shown in the interval $[0,1]$ and pattern \#3 values are shown in the interval $[0,-1]$, to indicate that the first has an up-then-down behavior, and the second has a down-then-up behavior. $(B)$ Number of promoters bound by Yap4 (left panel) or Sko1 (right panel) with the indicated binding time-course pattern for genes grouped according to their expression pattern. Colors denote fold enrichment relative to expected numbers, assuming random segregation of the groups. Asterisks indicate significance of the enrichment: $\left(^{\star}\right) P<0.05 ;\left(^{\star *}\right) P<1$ e- $6 .(C)$ Number of promoters bound by the indicated transcription factors at the indicated time after salt induction for genes grouped according to their expression time course pattern (top panel), their Yap4binding time-course pattern (middle panel), and Skol-binding time-course pattern (bottom panel). Colors denote fold enrichment relative to expected numbers, assuming random segregation of transcription factors among the groups. Asterisks indicate significance of the enrichment: $\left.\left.\right|^{\star}|P<0.05 ;|^{\star \star}\right) P<1 \mathrm{e}-6$.

negative regulation of gene expression by the same transcription factor has been previously noted for other factors (Martone et al. 2003; Theodorou et al. 2009).

We also examined the types of genes with different binding patterns and expression patterns. As shown in Supplemental Table S5, we found that several monosaccharide transporters exhibited induced Yap4 binding and induced expression, whereas a different set of transporters exhibited constitutive binding and repressed expression. Likewise, ribosomal protein genes were associated with Sknl binding and repressed expression. These results indicate that discrete types of genes are targeted, often in a temporal fashion to coordinate their expression.

The RNA expression of the different factors at the different time points was also examined (Supplemental Figure S4). Yap4 exhibited increased expression, consistent with the induced number of targets it bound, followed by a dip at $\sim 22-30 \mathrm{~min}$, perhaps due to autoregulation. For Skol and Skn7 a twofold to fourfold decrease in RNA expression was observed. Interestingly, most of the other factors did not change in RNA expression over the time course, indicating that their binding activity is likely regulated post-transcriptionally. Msn2 and Msn4 activity are regulated by nuclear localization. This is likely the case for many of the other factors as well.

Finally, to determine if any of the gene expression patterns were directly dependent on Yap4 and Yap6, we also analyzed RNA levels in cells deleted for YAP4 and YAP6. RNA was isolated from both untreated cells and those treated with $0.6 \mathrm{M}$ salt for $15 \mathrm{~min}, 30 \mathrm{~min}, 45 \mathrm{~min}$, and $60 \mathrm{~min}$, and hybridized to the tiling microarrays. Of 161 gene promoters bound by both Yap4 and Yap6, 47 displayed an alteration in gene expression pattern (data not shown). The expression pattern of the remaining genes was unchanged. These results indicate that the pattern of expression of a number of genes are dependent on Yap4 and/or Yap6. Other factors might contribute 
Ni et al.

to the expression pattern of the remainder, which is consistent with the hypothesis that the different factors may be redundant with one other for controlling gene expression.

\section{A dynamic transcription network controls the salt response of yeast}

The binding patterns of the different components allowed us to construct a regulatory network for the different factors relative to one another (Fig. 7A). We find a highly interconnected network whose connections change with time of induction. Skol is at the top of the network, and binds many other targets including Msn2, Msn4, Yap4, and Yap6 at both 0 min and $30 \mathrm{~min}$. Yap4, Yap6, Skn7, and Msn2 also bind many other regulators in the network. Interestingly, of all these, only Msn2 and Msn 4 binding is activated at $30 \mathrm{~min}$, and thus is presumably a key activator of the response. Of the downstream targets in the networks it is interesting to note that Msn4, Yap6, and Yap4 are targets of many other regulators (target hubs).

This network has many interesting feed-forward loops (e.g., Alon 2007). Msn2 is involved as an intermediate in three feed forward loops from Skol (to Yap4, Yap6, and Msn4). Given that Msn2 localize to the nucleus only after salt induction, while Skol binding changes very little after induction, these feed-forward loops up-regulate these three transcription factors in a Skol- and saltspecific manner. It is also interesting to note that the connection from Skol to Msn4 has two feed-forward loops involving Msn2 and Yap6 as intermediates, and that the connection from Skol to the intermediate connection Yap6 itself has a feed-forward connection involving Msn2. Such feed-forward loops have been suggested to serve as mechanisms to filter noise in environmental stimuli of gene expression. Thus, it would be interesting to investigate whether this arrangement of feed-forward loops enables Msn4-regulated genes to have a higher salt threshold for activation than either Msn2 or Yap6.

Each of these factors likely regulates many other genes. Figure 7B show that Msn1, Msn2, Skn7, Sko1, Yap4, and Yap6 bind a total of 88 other DNA-binding protein genes. Of these, SWI1, GAT1, BDF1, ROX1, HMS1, and GAT2 are each bound by five of the nine factors studied, and YOR315W, XBP1, TYE7, SUT2, SUT1, PHO1, NRG1, MSN4, IXR1, ASF1, SNF11, SFP1, NET1, MIG2, and CBF2
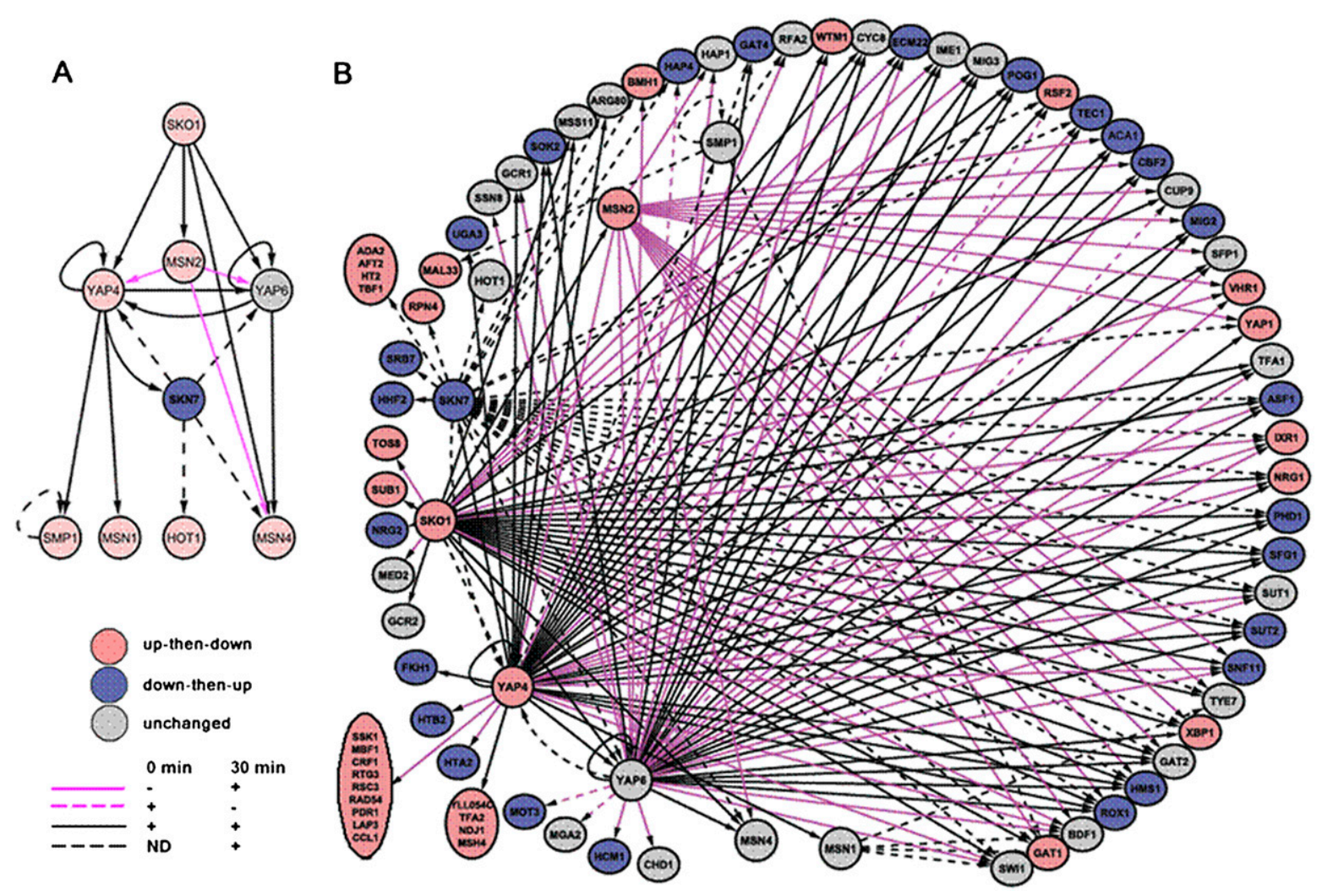

Figure 7. (A) Regulatory network for the nine transcription factors indicated as deduced from their binding to each others' promoters. (B) Regulatory network showing genes with the GO functional category "DNA-binding protein" and "Transcriptional regulatory protein" that are bound in their promoters by the transcriptional factors studied here (larger circles). Arrows point from the transcription factor to the gene whose promoter it binds. For clarity, a number of genes having the same edge type and source node are grouped inside ovals. (Pink solid arrows) Binding at $30 \mathrm{~min}$ post-induction only; (dashed pink arrow) binding at 0 min only; (solid black arrow) binding at both 0 min and $30 \mathrm{~min}$; (black dashed arrows) binding at $30 \mathrm{~min}$, undetermined at $0 \mathrm{~min}$. 
are each bound by four of them. Fifty-four transcription factor genes are bound by Yap4, 44 by Yap6, 34 by Sko1, and 30 by Skn7. Of the transcription factors that we studied, three, Yap4, Yap6, and Smp1, likely autoregulate as they bind their own promoters, while Yap4 and Yap6, and Skn7 and Yap4 bind to one another (Supplemental Figure S3). Thus, many regulators are themselves regulated by the salt response network.

Many of the connections are likely to be dynamic. For the transcription factors that we assayed at both $0 \mathrm{~min}$ and $30 \mathrm{~min}$ there were many more DNA-binding proteins bound at $30 \mathrm{~min}$ than at $0 \mathrm{~min}$. Besides the 43 distinct DNA-binding protein genes bound at both $0 \mathrm{~min}$ and $30 \mathrm{~min}$, there were five bound at only the 0 -min time point and 64 bound at only the 30 -min time point. Thus, many regulatory connections are induced during the response.

The different transcription factor targets are often key regulators of cellular processes. For example, Sfp1 and Crf1 are targets of Yap4 and are important for ribosomal protein synthesis, Rpn4 is a target of Skn 7 and involved in proteosome function, and Rtg3 is a target of Yap4 and regulates the Tor nutrient response pathway. These results indicate that the upstream factors not only directly bind to promoters of genes involved in cellular processes, but they also bind the regulators that control the same processes. Thus, a comprehensive control of cellular events is likely induced during the inductive response.

\section{Discussion}

In this study, we examined the dynamics of binding of key factors involved in a rapidly inducible process: the salt response of yeast. We examine two factors with complex patterns of binding: They are already bound at the initial time point, and exhibited increased numbers of binding sites at latter times (Fig. 2). A variety of binding patterns were revealed, including (1) constitutive binding (Yap4 and Sko1, Class 1), (2) rapid binding (Yap4 and Sko1, Class 2) (3) delayed binding (Yap4, Class 3), (4) transient binding (Sko1, Class 3; Yap4, minor Class 4), and reduced binding (Yap4, minor Class 5). These patterns indicate that even for individual factors complex patterns occur and define temporal classes of binding pattern used by eucaryotic regulators (Fig. 8). Correlation with targets bound by other factors that we also mapped revealed many associations among the different factors. In some cases (Yap4Yap6 and Smp1-Sko1) the coassociations were consistent with the binding patterns and with motif analysis, suggesting that the two factors cooperate to binding to their targets.

One important aspect of our study was to integrate binding and its dynamics with expression patterns. In many cases the association of individual factors correlated with expression patterns. For example, induction of rapid Yap4 binding (Class 2) at many targets correlates well with the induction of gene expression (Fig. 6B). However, many other interesting patterns also exist. For example, many constitutive targets (Class 1) bound

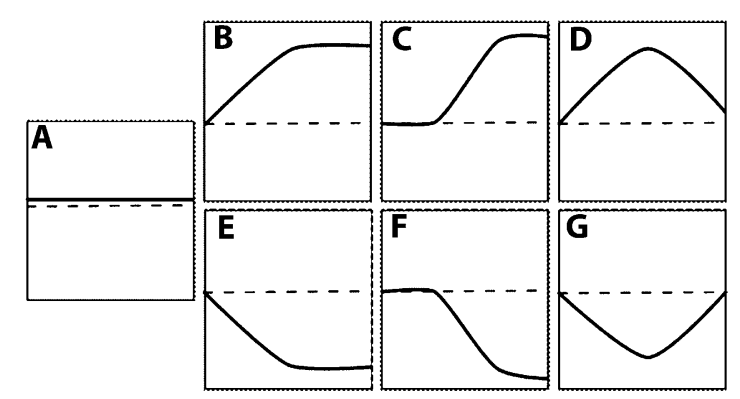

Figure 8. Types of temporal patterns of transcription factor binding. Patterns are based on those observed in Figure 2, as well as their equivalents with the opposite behavior (i.e., dissociation vs. binding). (A) Constitutive binding. (B) Rapid binding. $(C)$ Delayed binding. $(D)$ Transient binding. (E) Rapid dissociation. $(F)$ Delayed dissociation. (G) Transient dissociation.

by Yap4 and Yap6 actually exhibit a transient reduction in gene expression upon addition of salt. Presumably other parameters affect the outcome of these Yap4-bound targets, such as binding of other factors and modification of the proteins. Importantly, these results demonstrate that it is not only critical to find the gene targets, but the pattern of binding is likely to have an important role on the outcome. These results further demonstrate the importance of monitoring the dynamics of both binding and gene expression.

Many Yap4 and Skol targets are constitutively bound (Class 1). It is likely that other factors help regulate many of these genes. Consistent with this interpretation, Msn2 (which is not present at $0 \mathrm{~min}$ ) preferentially associates with Yap4 constitutive targets (Class 1) to activate their expression. Likewise, it is important to note that our analyses primarily focused on whether a target was bound or not. For many targets it is likely that the amount of binding in the promoter regions also changes and may mediate effects on gene expression.

Another important aspect of our study is that different combinations of factors appear to function together to regulate distinct sets of genes. For example, it appears that many factors (Yap4, Yap6, and Sko1) associate to regulate transporters. Thus, understanding the synergism among these different factors is crucial for understanding their functional role in a cell. Such information is vital for deciphering the regulatory code in eukaryotes. Indeed, for a complex process in which many cellular changes are regulated by a large number of factors it is essential to map the targets of many of these to fully understand the manner in which the suite of cellular events is regulated.

To help elucidate the specific combinations that preferentially associate to regulate genes set we require reasonable numbers of targets to attain statistical significance. One limitation with the approach used in this study is that many combinations target small number of genes, and thus their significance cannot be ascertained yet these associations are likely to be functional in many cases. Therefore, other approaches are important for directing ascertaining the function of these associations. 
In addition, it is likely that not all factors involved in the salt response have been analyzed (see motifs in Fig. 4) and thus, coassociations cannot be correlated in these cases. In the future it will also be useful to analyze factors such as Pho4, Sok2, Pdr3, Ume6, and other factors (e.g., Hal8 and $\mathrm{Hal9}$ ) to determine their role in mediating expression during this response (Mendizabal et al. 1998).

Our study provides insight into the regulation of regulatory network underlying an inducible response. A hierarchy was established with many dynamic steps and many constitutive connections. We found that many highly connected factors such as Sko1, Yap4, and Yap6 are constitutively bound at other regulators. In contrast, Msn2, which is normally cytosolic, is known to translocate to the nucleus under stress (Gorner et al. 1998; Mayordomo et al. 2002), resides at a key location in the network, and presumably is a key player in activation of the entire network.

Together, the control genes at the top of the network regulate a very large number of targets, both transcription factors and genes directly involved in cellular process. For example, Yap4 binds Sfp1, a regulator of ribosome biosynthesis, as well as directly binding promoters for ribosomal components. Why is the process so elaborate? Presumably, in a complex network such as the salt response, a large number of cellular processes are controlled such as metabolism, protein synthesis, and proteolysis, as evident by the gene expression changes. In order for cells to survive the process must be very rapid. Thus, direct regulation of the expression of components as well as their regulators, provides a comprehensive approach for regulating the cell state. Presumably other regulatory mechanisms such as protein modifications further control the state of the cell. In this respect many of the transcription factors analyzed such as Sko1, Smp1, Msn1, Msn2, and Msn4 are involved in Hog1-dependent responses that can regulate proteins by protein phosphorylation as well as transcriptionally (Proft et al. 2001; Proft and Struhl 2002; de Nadal et al. 2003; Hohmann et al. 2007).

We found previously that for a developmental response downstream, "targets hubs," which are bound by many factors, can serve as master regulators for inductive processes (Borneman et al. 2006). Interestingly, in the salt response network we found that overexpression of both upstream and downstream components (e.g., Skol, Yap4, Msn2, and Msn4) can activate resistance to salt, at least partially (X Zhu and M Snyder, unpubl.; data not shown). We speculate that in a rapid defense response such as exposure to toxic conditions, it is critical to have upstream components quickly activating biological responses for cell survival. In contrast, a developmental response, which leads to a major and sometimes irreversible commitment, presumably reads many upstream signals before committing to a response through a master regulator. Finally, we note that the salt response and Hog1 pathways are highly conserved among eucaryotes (Hohmann 2002); therefore, we expect that the principles learned in this study will be generally applicable to many organisms.

\section{Materials and methods}

\section{Yeast strains and growth conditions}

S. cerevisiae strains were all derived from BY4741 (leu2dO

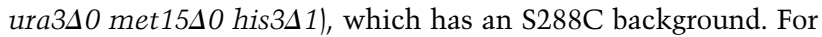
the induction of salt response, $S$. cerevisiae strains were first grown overnight to mid-exponential phase (OD $600=0.6)$ at $30^{\circ} \mathrm{C}$ in liquid minimal medium $(\mathrm{SD} ; 2 \%$ glucose, $0.5 \% 38 \mathrm{mM}$ ammonium sulphate, $0.17 \%$ yeast nitrogen base, $5 \mathrm{mM}$ uracil), then treated with $0.6 \mathrm{M} \mathrm{NaCl}$ (generated using a $5 \mathrm{M}$ stock solution) at $30^{\circ} \mathrm{C}$. Samples were then harvested by vacuum filtration at different time points. For the time course of ChIP chip analysis, yeast cells were collected at $0,5,15,30$, and $45 \mathrm{~min}$ after salt induction, for gene expression analysis, yeast cells were collected at $0,15,22,30,45$, and 60 min after salt induction.

\section{Gene deletion, epitope tagging, and Western blotting}

$S$. cerevisiae genes were epitope-tagged using the long-primer PCR approach and the plasmid pFA6a-13myc-KanMX as a template (Lorenz et al. 1995; Longtine et al. 1998). PCR products were transformed into the haploid strains BY4741 using a lithium acetate (LiAc) technique (Gietz and Woods 2002). Transformants were selected by growth on YPD plates containing $200 \mathrm{mg} / \mathrm{mL}$ G418. Strains were tested for correct integration by PCR. Western blots were performed on protein lysates prepared from induced (see above) cells. Following SDS-PAGE and transfer to nitrocellulose, fusion proteins were detected using anti-myc antibody (monoclonal 9E10, 1:2000 dilution) and visualized by chemiluminescence. Although the kinetics of binding could be affected by the epitope tags, the tagged proteins appeared to be functional as evident by resistance to salt treatment, similar to wild-type cells.

\section{ChIP DNA labeling and array hybridizations}

ChIPs and DNA labeling were performed as described previously (Borneman et al. 2006). Hybridizations were carried out according to manufacturer's protocols (Nimblegen LLC) using customdesigned microarrays (Borneman et al. 2006). Chip chip data has been deposited at the NCBI GEO site with the accession GSE15639 and at http://archive.gersteinlab.org/proj/yeast_salt. A list of targets are present in Supplemental Table S3.

\section{Expression array hybridizations}

Total RNA was extracted from Ribopure Yeast kit (Ambion) and treated for $30 \mathrm{~min}$ at $37^{\circ} \mathrm{C}$ with RNase-free DNase I (Ambion). Poly(A) RNA was purified with Micro Poly(A) purist kit according to the manufacturer's instructions (Ambion). Dye-labeled cDNA was prepared from $1 \mu \mathrm{g}$ of poly(A) RNA (amino-allyl cDNA labeling kit, Ambion) and hybridized to microarrays that were spotted with 70-mer oligonucleotide probes representing all of the ORFs of $S$. cerevisiae (Yeast AROS version 1.1, Qiagen). Gene expression lists are presented in Supplemental Table S4. The hybridization data are available at http://archive.gersteinlab. org/proj/yeast_salt.

\section{PCR validation of binding}

PCR validation of the ChIP chip results were performed as described previously (Borneman et al. 2006). qPCR was performed using a Roche LightCycler 480. Fold enrichment of tagged versus untagged signal was calculated for each primer pair; for these measurements the average level of enrichment 
observed with three control (i.e., nontarget regions) was subtracted to correct for small differences in the level of input DNA. Each GPCR value presented represents the average of three independent measurements of fold enrichment for each intergenic region.

\section{Data processing and analysis}

Expression slides were scanned using a Genepix 4000A scanner (Axon Instruments) and analyzed using Genepix 3.0 (Axon Instruments). Following scanning, Genepix result (.gpr) files were uploaded to the Express Yourself Web site (http://array. mbb.yale.edu/analysis) (Luscombe et al. 2003) for normalization.

Tiling data was obtained with custom-designed microarrays as described in Borneman et al. (2006). Tiling array data was preprocessed in the following manner. First, data quality on the microarray was checked for any gross imperfections or abnormal signal heterogeneities. Spatial artifacts were removed by normalizing each probe's value to the mean of a $25 \times 25$ feature square surrounding it. In order to avoid artificially high values due to cross-hybridization by highly homologous sequences from other parts of the genome, probes belonging to sequence repeats (telomere sequences, LTRs, rRNA genes, transposons) were removed. Next, data from three biological replicates were combined by quantile normalization and averaged across a moving window of 10 probes, as described in Royce et al. (2006).

The logarithm of the red/green signal ChIP chip data was assumed to be the summation of a "background" component and a specific signal. The background component was assumed to be symmetrical and centered at zero, while the specific signal was only positive. Thus, all data was normalized by shifting the histogram of the signal values so its mode was at zero and scaled so the left flank of its histogram corresponded to a standard deviation of 0.1 . A threshold corresponding to false discovery rate $(F D R)=0.01$, was selected such that the ratio of the area in the histogram to the right of the threshold value for the assumed null signal distribution to that of the specific signal distribution was equal to 0.01 . The signal histogram for Msn2 and Msn4 at $0 \mathrm{~min}$, when these two transcription factors are not localized to the nucleus and therefore unable to bind DNA /Gorner et al. 1998; Mayordomo et al. 2002), was nearly symmetrical, validating this method of subtracting the background component of the signal (Supplemental Fig. S1).

In the case of Yap4 and Sko1, for which binding data was obtained for five time points, the data for the different time points was normalized using the qPCR data. In the qPCR experiment, 42 genomic regions of about 200 -nucleotide (nt) size were assayed on the same day for all five time points by amplification by qPCR after ChIP. By plotting the signal from qPCR and ChIP chip, the data from the two methods were found to be tightly correlated $(r>0.90)$. The ChIP chip data was calibrated against the qPCR data, by plotting each ChIP chip data set against its corresponding qPCR data, after shifting the qPCR data so the regression line went through the origin. Then all ChIP chip data was multiplied with a correction factor that equalized the slopes of the calibration curves to their average.

Peaks were mapped from the signal map at where the slope of a regression line inside a 25-probe moving window shifted sign (i.e., went through zero). The signal value of the peak was taken as the average of a 400 base stretch centered at the peak point. To create a high-confidence time-course data set for Yap4 and Sko1, only the target regions that had above-threshold values in two consecutive time points after induction were used. Missing data for such targets were filled in with the measured signal even if below threshold.
In assigning a binding site to a promoter, we arbitrarily defined the promoter region of a gene to start at either the boundary of its upstream ORF or $1000 \mathrm{bp}$ upstream of the beginning of its own ORF, whichever is nearer, and end at $50 \mathrm{nt}$ inside the ORF. It was noteworthy that the number of binding sites that fell within the promoters ranged from $\sim 81 \%$ to $22 \%$ (Supplemental Table S1). The transcription factors bound at the promoters of ORFs and their expression levels relative to time $=0 \mathrm{~min}$ are listed in Supplemental Table S6.

\section{Clustering of the binding time courses}

Clustering of the binding time courses was performed by hierarchical clustering, after rescaling the data to a maximum of 1 , and using 1 minus correlation coefficient as a distance metric. The resultant clusters were similar to those obtained by clustering using the portioning around medoids (PAM) algorithm (Kaufman and Rousseeuw 1990). The optimal number of clusters was determined by the silhouette method (Kaufman and Rousseeuw 1990). For clustering expression data, first, the signal that was unchanging across time (maximum minus minimum $<0.5 \log 2$ units) was removed, then the remaining signal was normalized by setting the maximum to 1 and the minimum to 0 .

\section{GO enrichment and motif analysis}

GO analysis was conducted with the GO Term Finder service at the Sacchromyces Genome Database (http://www.yeastgenome. org), using as the background set only ORFs of status "uncharacterized" or "verified."

Motif analysis was performed with MEME, MDscan, and Bioprospector (Bailey and Elkan 1994; Liu et al. 2001, 2002). The identified motifs were assigned to transcription factors using the database of yeast transcription factors based on MacIsaac et al. (2006), Badis et al. (2008), and Zhu et al. (2009), using the Web service STAMP (Mahony and Benos 2007). Network diagrams were generated with Cytoscape (Shannon et al. 2003).

The significance of the number of overlapping elements in two lists of genes was estimated by using the accumulative distribution of hypergeometric distribution, using the phyper function in $\mathrm{R}: P$-value $=\operatorname{phyper}[\min (n 1, n 2), n 1, n-n 1, n 2]-\operatorname{phyper}(m-1$, $n 1, n-n 1, n 2)$, where $n$ is the total number of genes, $n 1$ is the number of genes in one list, $n 2$ is the number of genes in the second list, and $m$ is the number in the overlap between the two lists.

\section{Acknowledgments}

We thank Xiuqiong Zhou for yeast culture work. The work from M.S.'s laboratory was funded by grants from the NIH. C.B. was funded by NIH grant T15LM07056 from the National Library of Medicine.

\section{References}

Alon U. 2007. Network motifs: Theory and experimental approaches. Nat Rev Genet 8: 450-461.

Badis G, Chan ET, van Bakel H, Pena-Castillo L, Tillo D, Tsui K, Carlson CD, Gossett AJ, Hasinoff MJ, Warren CL, et al. 2008. A library of yeast transcription factor motifs reveals a widespread function for Rsc3 in targeting nucleosome exclusion at promoters. Mol Cell 32: 878-887.

Bailey TL, Elkan C. 1994. Fitting a mixture model by expectation maximization to discover motifs in biopolymers. Proc Int Conf Intell Syst Mol Biol 2: 28-36. 
Berry DB, Gasch AP. 2008. Stress-activated genomic expression changes serve a preparative role for impending stress in yeast. Mol Biol Cell 19: 4580-4587.

Borneman AR, Leigh-Bell JA, Yu H, Bertone P, Gerstein M, Snyder M. 2006. Target hub proteins serve as master regulators of development in yeast. Genes \& Dev 20: 435-448.

Borneman AR, Gianoulis TA, Zhang ZD, Yu H, Rozowsky J, Seringhaus MR, Wang LY, Gerstein M, Snyder M. 2007a. Divergence of transcription factor binding sites across related yeast species. Science 317: 815-819.

Borneman AR, Zhang ZD, Rozowsky J, Seringhaus MR, Gerstein M, Snyder M. 2007b. Transcription factor binding site identification in yeast: A comparison of high-density oligonucleotide and PCR-based microarray platforms. Funct Integr Genomics 7: 335-345.

Chi Y, Huddleston MJ, Zhang X, Young RA, Annan RS, Carr SA, Deshaies RJ. 2001. Negative regulation of Gen4 and Msn2 transcription factors by Srb10 cyclin-dependent kinase. Genes \& Dev 15: 1078-1092.

de Nadal E, Casadome L, Posas F. 2003. Targeting the MEF2-like transcription factor Smp1 by the stress-activated Hog1 mitogen-activated protein kinase. Mol Cell Biol 23: 229-237.

Gasch AP, Spellman PT, Kao CM, Carmel-Harel O, Eisen MB, Storz G, Botstein D, Brown PO. 2000. Genomic expression programs in the response of yeast cells to environmental changes. Mol Biol Cell 11: 4241-4257.

Gietz RD, Woods RA. 2002. Transformation of yeast by lithium acetate/single-stranded carrier DNA/polyethylene glycol method. Methods Enzymol 350: 87-96.

Gorner W, Durchschlag E, Martinez-Pastor MT, Estruch F, Ammerer G, Hamilton B, Ruis H, Schuller C. 1998. Nuclear localization of the $\mathrm{C} 2 \mathrm{H} 2$ zinc finger protein $\mathrm{Msn} 2 \mathrm{p}$ is regulated by stress and protein kinase A activity. Genes \& Dev 12: 586-597.

Hamatani T, Carter MG, Sharov AA, Ko MS. 2004. Dynamics of global gene expression changes during mouse preimplantation development. Dev Cell 6: 117-131.

Harbison CT, Gordon DB, Lee TI, Rinaldi NJ, Macisaac KD, Danford TW, Hannett NM, Tagne JB, Reynolds DB, Yoo J, et al. 2004. Transcriptional regulatory code of a eukaryotic genome. Nature 431: 99-104.

Hohmann S. 2002. Osmotic stress signaling and osmoadaptation in yeasts. Microbiol Mol Biol Rev 66: 300-372.

Hohmann S, Krantz M, Nordlander B. 2007. Yeast osmoregulation. Methods Enzymol 428: 29-45.

Horak CE, Luscombe NM, Qian J, Bertone P, Piccirrillo S, Gerstein M, Snyder M. 2002. Complex transcriptional circuitry at the G1/S transition in Saccharomyces cerevisiae. Genes \& Dev 16: 3017-3033.

Jakobsen JS, Braun M, Astorga J, Gustafson EH, Sandmann T, Karzynski M, Carlsson P, Furlong EE. 2007. Temporal ChIPon-chip reveals Biniou as a universal regulator of the visceral muscle transcriptional network. Genes \& Dev 21: 24482460.

Kaufman L, Rousseeuw PJ. 1990. Finding groups in data: An introduction to cluster analysis. Wiley, New York.

Lam FH, Steger DJ, O'Shea EK. 2008. Chromatin decouples promoter threshold from dynamic range. Nature 453: 246250.

Liu X, Brutlag DL, Liu JS. 2001. BioProspector: Discovering conserved DNA motifs in upstream regulatory regions of coexpressed genes. Pac Symp Biocomput 6: 127-138.

Liu XS, Brutlag DL, Liu JS. 2002. An algorithm for finding protein-DNA binding sites with applications to chromatinimmunoprecipitation microarray experiments. Nat Biotechnol 20: 835-839.
Longtine MS, McKenzie A III, Demarini DJ, Shah NG, Wach A, Brachat A, Philippsen P, Pringle JR. 1998. Additional modules for versatile and economical PCR-based gene deletion and modification in Saccharomyces cerevisiae. Yeast 14: 953961.

Lorenz MC, Muir RS, Lim E, McElver J, Weber SC, Heitman J. 1995. Gene disruption with PCR products in Saccharomyces cerevisiae. Gene 158: 113-117.

Luscombe NM, Royce TE, Bertone P, Echols N, Horak CE, Chang JT, Snyder M, Gerstein M. 2003. ExpressYourself: A modular platform for processing and visualizing microarray data. Nucleic Acids Res 31: 3477-3482.

Luscombe NM, Babu MM, Yu H, Snyder M, Teichmann SA, Gerstein M. 2004. Genomic analysis of regulatory network dynamics reveals large topological changes. Nature 431: 308312.

MacIsaac KD, Wang T, Gordon DB, Gifford DK, Stormo GD, Fraenkel E. 2006. An improved map of conserved regulatory sites for Saccharomyces cerevisiae. BMC Bioinformatics 7: 113. doi: 10.1186/1471-2105-7-113.

Mahony S, Benos PV. 2007. STAMP: A Web tool for exploring DNA-binding motif similarities. Nucleic Acids Res 35: W253-W258. doi: 10.1093/nar/gkm272.

Martone R, Euskirchen G, Bertone P, Hartman S, Royce TE, Luscombe NM, Rinn JL, Nelson FK, Miller P, Gerstein M, et al. 2003. Distribution of NF-кB-binding sites across human chromosome 22. Proc Natl Acad Sci 100: 1224712252.

Mayordomo I, Estruch F, Sanz P. 2002. Convergence of the target of rapamycin and the Snf1 protein kinase pathways in the regulation of the subcellular localization of Msn2, a transcriptional activator of STRE (stress response element)-regulated genes. J Biol Chem 277: 35650-35656.

Mendizabal I, Rios G, Mulet JM, Serrano R, de Larrinoa IF. 1998. Yeast putative transcription factors involved in salt tolerance. FEBS Lett 425: 323-328.

Nevitt T, Pereira I, Azevedo D, Guerreiro P, Rodrigues-Pousada C. 2004. Expression of YAP4 in Saccharomyces cerevisiae under osmotic stress. Biochem J 379: 367-374.

Pascual-Ahuir A, Posas F, Serrano R, Proft M. 2001. Multiple levels of control regulate the yeast cAMP-response elementbinding protein repressor Skolp in response to stress. I Biol Chem 276: 37373-37378.

Proft M, Struhl K. 2002. Hog1 kinase converts the Sko1-Cyc8Tup1 repressor complex into an activator that recruits SAGA and SWI/SNF in response to osmotic stress. Mol Cell 9: 1307-1317.

Proft M, Pascual-Ahuir A, de Nadal E, Arino J, Serrano R, Posas F. 2001. Regulation of the Sko1 transcriptional repressor by the Hog 1 MAP kinase in response to osmotic stress. EMBO J 20: $1123-1133$.

Proft M, Gibbons FD, Copeland M, Roth FP, Struhl K. 2005. Genomewide identification of Skol target promoters reveals a regulatory network that operates in response to osmotic stress in Saccharomyces cerevisiae. Eukaryot Cell 4: 13431352.

Reed BD, Charos AE, Szekely AM, Weissman SM, Snyder M. 2008. Genome-wide occupancy of SREBP1 and its partners NFY and SP1 reveals novel functional roles and combinatorial regulation of distinct classes of genes. PLoS Genet 4: e1000133. doi: 10.1371/journal.pgen.1000133.

Rep M, Reiser V, Gartner U, Thevelein JM, Hohmann S, Ammerer G, Ruis H. 1999. Osmotic stress-induced gene expression in Saccharomyces cerevisiae requires Msnlp and the novel nuclear factor Hotlp. Mol Cell Biol 19: 5474-5485. 
Royce TE, Rozowsky JS, Luscombe NM, Emanuelsson O, Yu H, Zhu X, Snyder M, Gerstein MB. 2006. Extrapolating traditional DNA microarray statistics to tiling and protein microarray technologies. Methods Enzymol 411: 282-311.

Shannon P, Markiel A, Ozier O, Baliga NS, Wang JT, Ramage D, Amin N, Schwikowski B, Ideker T. 2003. Cytoscape: A software environment for integrated models of biomolecular interaction networks. Genome Res 13: 2498-2504.

Simon I, Barnett J, Hannett N, Harbison CT, Rinaldi NJ, Volkert TL, Wyrick JJ, Zeitlinger J, Gifford DK, Jaakkola TS, et al. 2001. Serial regulation of transcriptional regulators in the yeast cell cycle. Cell 106: 697-708.

Tan K, Feizi H, Luo C, Fan SH, Ravasi T, Ideker TG. 2008. A systems approach to delineate functions of paralogous transcription factors: Role of the Yap family in the DNA damage response. Proc Natl Acad Sci 105: 2934-2939.

Theodorou E, Dalembert G, Heffelfinger C, White E, Weissman S, Corcoran L, Snyder M. 2009. A high throughput embryonic stem cell screen identifies Oct-2 as a bifunctional regulator of neuronal differentiation. Genes \& Dev 23: 575588.

Tseng GC. 2007. Penalized and weighted K-means for clustering with scattered objects and prior information in high-throughput biological data. Bioinformatics 23: 2247-2255.

Zahringer H, Thevelein JM, Nwaka S. 2000. Induction of neutral trehalase Nth1 by heat and osmotic stress is controlled by STRE elements and Msn2/Msn4 transcription factors: Variations of PKA effect during stress and growth. Mol Microbiol 35: 397-406.

Zhang ZD, Rozowsky J, Lam HY, Du J, Snyder M, Gerstein M. 2007. Tilescope: Online analysis pipeline for high-density tiling microarray data. Genome Biol 8: R81. doi: 10.1186/gb2007-8-5-r81.

Zhu C, Byers KJ, McCord RP, Shi Z, Berger MF, Newburger DE, Saulrieta K, Smith Z, Shah MV, Radhakrishnan M, et al. 2009. High-resolution DNA-binding specificity analysis of yeast transcription factors. Genome Res 19: 556-566. 


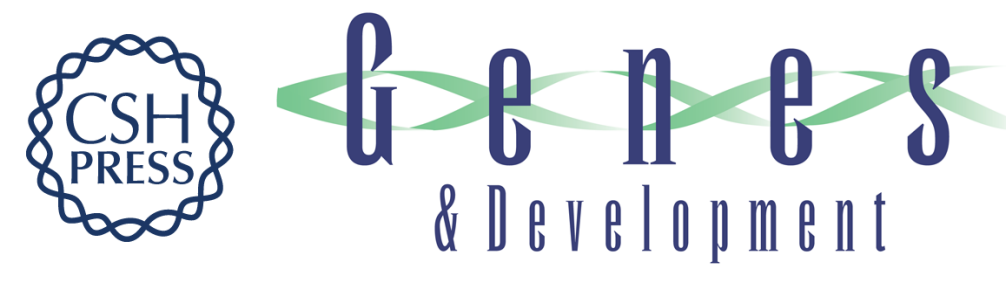

\section{Dynamic and complex transcription factor binding during an inducible response in yeast}

Li Ni, Can Bruce, Christopher Hart, et al.

Genes Dev. 2009, 23:

Access the most recent version at doi:10.1101/gad.1781909

Supplemental http://genesdev.cshlp.org/content/suppl/2009/05/20/23.11.1351.DC1
Material

References This article cites 46 articles, 20 of which can be accessed free at: http://genesdev.cshlp.org/content/23/11/1351.full.html\#ref-list-1

License

Email Alerting

Service

Receive free email alerts when new articles cite this article - sign up in the box at the top right corner of the article or click here.

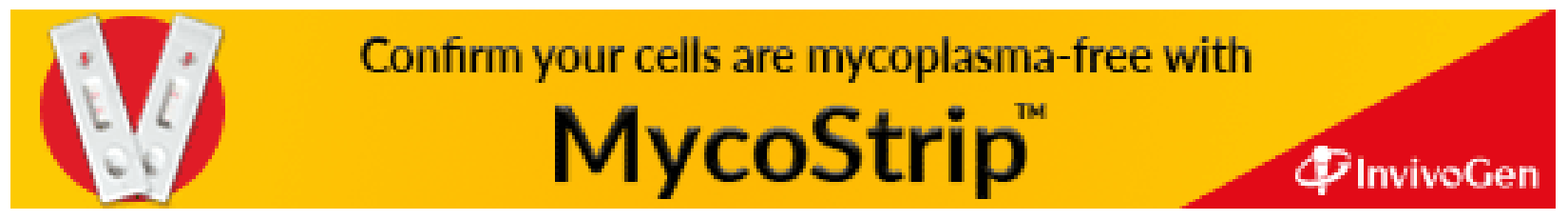

